\title{
Distributed Coordination Schemes for Multi- Radio Co-existence in Dense Spectrum Environments: An Experimental Study on the ORBIT Testbed
}

\author{
Xiangpeng Jing, Shanmuga S Anandaraman, Mesut Ali Ergin, Ivan Seskar, Dipankar Raychaudhuri \\ WINLAB, Rutgers University \\ 671 Route 1 South \\ North Brunswick, NJ 08902 \\ \{xjing, shanmuga, ergin, seskar, ray\}@winlab.rutgers.edu
}

\begin{abstract}
This paper presents an experimental study on the spectrum coexistence ${ }^{1}$ problems between multi-radio platforms in dense-radio physical world environments. Computing and communication devices such as laptops and cellular phones with multiple radios including WiFi, Bluetooth, UWB, WiMax and Zigbee in a small conference room face significant interference problems. A realistic small office/home office (SOHO) scenario with $\sim 10-25$ multi-radio platforms is mapped onto the ORBIT radio grid testbed, and system throughput results are obtained experimentally, demonstrating significant degradation due to inter-platform interference. The CSCC (Common Spectrum Coordination Channel) protocol proposed in earlier work is used as the basis for implementing a set of distributed spectrum coexistence algorithms intended to improve system performance. Detailed results from ORBIT testbed experiments are given for the proposed CSCC-based distributed spectrum coordination algorithms. The results show significant performance gains due to $\mathrm{CSCC}$ coordination, typically achieving $\sim 2 \mathrm{x}$ improvement in system throughput for $\mathrm{WiFi} / \mathrm{Bluetooth}$ dual radio scenarios.
\end{abstract}

Keywords-Multi-radio coexistence; Distributed spectrum coordination; CSCC; ORBIT

\section{INTRODUCTION}

Multi-radio portable computing platforms such as laptops and PDAs integrated with many different radios (e.g. WiFi, WiMax, UWB, Bluetooth, DVB, GPS) are becoming the norm these days. Proliferation of these devices will result in dense spectrum usage patterns with as many as $\sim 10$ 's of radios per square meter operating in a variety of licensed and unlicensed bands. The technical challenge associated with the emerging multi-radio scenario is that of defining a framework for distributed coordination between nearby devices with the objective of achieving good application performance with high spectrum efficiency. Clearly, this is a complex distributed control problem for which the general solution involves a combination of several techniques, including

\footnotetext{
${ }^{1}$ Research supported by Intel Corp and NSF grant CNS-0626740.
}

spectrum sensing and power control, dynamic spectrum sharing, and medium access control (MAC) layer coordination.

Simple reactive spectrum coordination methods [1] may be used in multi-radio networks where radio nodes react to interference and channel condition changes by tuning their runtime radio parameters such as operating channel, transmit power, rate, etc. to avoid interference. All these schemes have a low level of complexity since no extra equipment is needed but they have limited performance especially in dense scenarios with hidden nodes as highlighted in [2]. In the multi-radio environment, heterogeneous radio technologies can share part of the spectrum and the interference problems can be categorized into two different kinds: the interference due to co-located radios in the same platform (due to close antennas in a physical separation of only inches); and the interference due to close proximity of different wireless platforms (due to close locations of devices in a physical separation of several feet). Usually the interference problem is quite complicated in such a dense multi-radio scenario and it is not easy for simple reactive methods to find the gaps between spectrum-use bursts for interference avoidance.

For improved performance, the concept of protocolassisted coordination between radio nodes has been investigated through this work, leading to coordination schemes based on the Common Spectrum Coordination Channel (CSCC) [3]. Spectrum and radio usage information are exchanged via CSCC messages which contain information such as node ID, radio type, center frequency, bandwidth, transmit power, data rate, modulation type, service type, etc. Thus radio nodes can explicitly execute coordination algorithms and adapt their transmit parameters by using appropriate distributed algorithms [4]. CSCC-based protocols have been 
previously applied to coexistence scenarios of standalone IEEE 802.11 and Bluetooth [5-8], and also IEEE 802.11 with IEEE 802.16 [1][4]. In this work, we apply the CSCC-based protocol to the dense multi-radio scenarios and focus on designing specific interference adaptation algorithms which can help heterogeneous radios to coordinate their operating parameters and reduce interference. In particular, we evaluate these spectrum coordination algorithms in scenarios with densely distributed IEEE $802.11 \mathrm{~g}$ and Bluetooth dual-radio platforms for a typical small office home office (SOHO) scenario, and experiment on a realistic setup using the ORBIT testbed [14]. We start with Bluetooth avoidance algorithm (Bo) which adapt Bluetooth transfer time and bit-rate and can provide a significant performance gain in IEEE $802.11 \mathrm{~g}$ throughput. Then we study more complex algorithms for more balanced system throughput and these include Bluetooth rate-adaptation (Rt) and SIR-based adaptation.

The paper is divided into the following sections. In Sec II, we discuss spectrum coexistence problems in multi-radio scenarios and explain how protocol-assisted algorithms can be used to improve performance. Sec III provides a detailed discussion of the spectrum coordination algorithms proposed for the CSCC-based protocol. In Sec IV, we provide experimental evaluation results for the algorithms proposed. Finally, concluding remarks are given in Sec V.

\section{SPECTRUM COORDINATION IN MULTI-RADIO NETWORKS}

\section{A. Multi-radio Scenario}

In our study, we characterize typical usage scenarios in multi-radio co-existence environments by identifying representative habitats such as home, small/home office (SOHO), and large enterprise. These habitats are considered on appropriate floor-plans which home the platforms with various radio technologies to reflect their role in occupant's lives by their quantity, real-world physical placement and offered traffic.

In this paper, due to space considerations, we use the SOHO scenario as a representative basis for our analysis. This scenario has devices equipped with multiple radios including WiFi, Bluetooth, ZigBee, WiMax and UWB. Fig. 1 illustrates the baseline SOHO scenario with about 20 active people working in a semi-partitioned dry-wall space of about $20 \mathrm{~m} \mathrm{x}$ $20 \mathrm{~m}$. The habitat has one active main conference room (on the left, middle), a smaller meeting room (far upper corner), as well as office, cubicle and lab spaces (on the center and rightmost side). Laptops with $\mathrm{WiFi}$ and Bluetooth radios are clustered in the conference room and distributed in cubicle areas and we use their topology in our experimental evaluations given in Section IV. The conference room is also equipped with an UWB projector for laptop-to-wall streaming. WiMax-equipped handhelds or laptops are also used for wide-area access by the visitors. Sensors with ZigBee radios are distributed throughout the habitat and communicate for the smart building infrastructure.

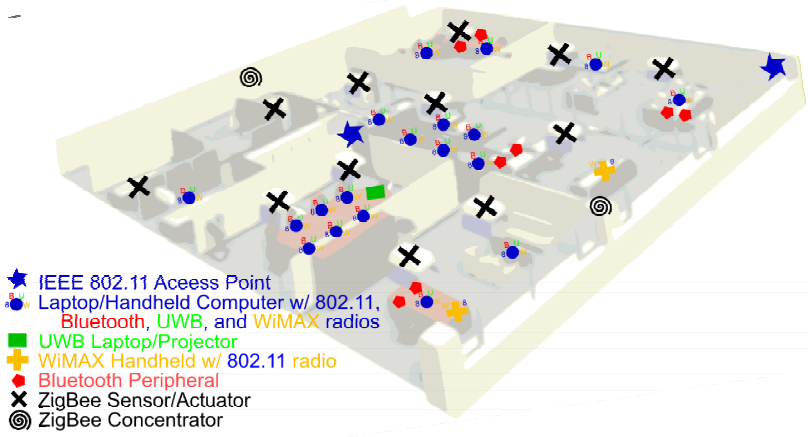

Figure 1. SOHO scenario for multi-radio co-existence.

The WiFi network for this office (about 20 people) can be supported by two access points where one of them is closer to the central area of activity, the conference room and the other is on the rightmost corner of the floor plan. The typical workload for the WiFi network is obtained from an observation study of a similar office $\mathrm{WiFi}$ network used in [13]. In summary, 97\% of workload is composed of TCP traffic (remaining 3\% being UDP for VoIP communications), which further decomposed as $75 \%$ WWW browsing over HTTP, and $25 \%$ other background traffic (such as NETBIOS, FTP and SSH file transfers, printing, etc.) The Bluetooth radios in this scenario are mostly used for cell-phone-to-headset and laptop-to-headset audio streaming. It is appropriate to consider this workload as point-to-point CBR-type streams with different service levels corresponding to different audio CODECs requiring $64 \mathrm{Kbps}, 128 \mathrm{Kbps}, 256 \mathrm{Kbps}$, and $320 \mathrm{Kbps}$.

Also, WiMax traffic is considered to carry VoIP data using $96 \mathrm{Kbps}$ UDP streams of 300-byte frames, tolerating a maximum of $5 \%$ loss with $30 \mathrm{~ms}$ delay requirement. The smart building infrastructure uses ZigBee sensors/actuators on each light fixture as well as climate sensors and the two ZigBee concentrators act as relays for the communication to the rest of the infrastructure in the building. The sensor traffic is assumed to be sub-minute readings of 50-byte frame updates and occasional actuation communications not exceeding a couple of Kbps.

In the first stage of our evaluation study, we map the WiFi and Bluetooth part of the outlined SOHO scenario on to the ORBIT testbed with IEEE $802.11 \mathrm{a} / \mathrm{g}$ and Bluetooth ver1.2 dual-radio nodes. While this serves as a starting point for evaluating our spectrum coordination protocols and algorithms, we will augment in the future the ORBIT testbed with WiMax, Zigbee, and UWB radios to enable more complete evaluation of co-existence.

\section{B. Interference Problems in Multi-radio Platforms}

Radio co-existence/interference problems have been studied widely in scenarios where wireless devices with 
different radio technologies share the same spectrum band, typically the $2.4 \mathrm{GHz}$ ISM band. Examples of these studies include the coexistence of IEEE 802.11b with Bluetooth [7$11]$, and the coexistence of IEEE $802.11 \mathrm{~b}$ with WiMax operating in the same unlicensed band [1][4]. For these scenarios, single-radio devices are assumed to be randomly distributed in the same physical area and since these devices use different physical layer technologies, they are not able to decode each others' frames. Such unawareness translates to uncoordinated transmissions and large amount of frame losses due to proximity-dependent interference, when transmissions overlap both in time and frequency domain ${ }^{2}$. We refer to this type of interference as inter-node interference.

In multi-radio environments, the only source of the interference may not be the other wireless devices lying around. With the increasing number of radios per platform (i.e., laptop, PDA, or smart-phone), limited device size and insufficient antenna isolation create significant interference problems, as well. Interference effects are more severe when multiple radios share a co-located antenna and possibly other RF components. The worst-case scenario of interference within the same platform is likely to be the concurrent transmission from one of the higher-power radios of the platform while another one is in the process of reception. We refer to this type of interference as intra-node interference or in-platform interference. When considered with other kinds of interference inherent to the platform (e.g., harmonics from CPU, GPU and other platform clocks, cross-modulation through common power sources) intra-node interference and inter-node interference pose an undeniable threat to the operation of densely deployed multiple radios in harmony.

The interference problems due to co-located radios of different bands can largely be addressed by implementing improved antenna isolation and better filters early in the radio receiver chains. However, enforcing higher degrees of antenna isolation becomes a challenge in space-limited platforms and better filters are usually avoided in practice because of their prohibitive costs. Such physical layer approaches are also static in nature and do not fit in a dynamically varying number/type of multi-radio scenarios, including software radios. Another typical solution for interference mitigation is achieved by scheduling radio transmissions to serialize interference-causing actions in time. Signaling required for this approach is easier to implement inplatform. Hardware vendors may collaborate for tight-control of radios through designated hardware buses. Also courselevel control is possible through software-scheduling implemented within the OS and radio drivers which coordinate radio transmission and receiving patterns in a time-sharing manner. This TDM-type scheduling approach can partly prevent intra-node interference; however serialization is a low efficiency operation that reduces throughput. More importantly, TDM-type scheduling involves

\footnotetext{
${ }^{2}$ Note that this is not the only reason inter-node interference is observed in practice. Transmit power differences coupled with imperfections in receiver front-ends are also significant sources of interference.
}

high overhead and might fail on occasions where utilization of one radio is close to $100 \%$ during its session with no scope of time sharing the channel. In the remainder of the paper, we propose and implement a higher-level radio-independent Multi-Radio Coexistence and Coordination approach that aims to provide a scalable solution to various types of multi-radio setups, similar in purpose to the MICE, proposed in [2]. A set of high level distributed coordination protocols is developed on top of CSCC (Common Spectrum Coordination Channel) based spectrum etiquette protocols, proposed in our previous work [3]. Results indicate efficient mitigation of multiradio inter-node and intra-node interference and improvements in application level performance, as detailed in Section IV.

\section{CSCC-based Spectrum Coordination Protocols}

When dense multi-radio scenarios possibly with hidden nodes are considered, simple reactive spectrum coordination methods (i.e., adjusting transmission parameters after interference degrades the performance) or in-platform scheduling algorithms (i.e., assuming the only interference source is the other radios of the same device) usually have limited performance and value. Lack of a global view of the radio network is to blame for the limited performance of such approaches. For improved performance, the concept of protocol-assisted coordination between radio nodes has been investigated in [5-6], leading to a specific scheme called the Common Spectrum Coordination Channel (CSCC) [3]. As shown in Fig. 2, each wireless node in the environment uses a common control radio standard, operating at a known frequency for control and coordination purposes.

The CSCC protocol is based on a common signaling mechanism, which can be implemented either using a common control channel at the edge of the shared spectrum, or in a control mode (time slot) where the same radio can periodically switch back and forth between control and regular communication states. In the example of Fig. 2, the CSCC protocol uses Channel 1 of the $2.4 \mathrm{GHz}$ ISM Band for 1 Mbps IEEE $802.11 \mathrm{~b}$ communication as the basis - in a multi-radio system, this means that each platform must be capable of periodically tuning to the specified CSCC channel to obtain control information. Spectrum and radio usage information are exchanged via CSCC messages which contain information such as node ID, radio type, center frequency, bandwidth, transmit power, data rate, modulation type, service type, etc. It is also possible for radio nodes to aggregate information received from other neighboring radios and forward them to create a global awareness of radio resource usage among nearby platforms. Thus, radio nodes can explicitly execute coordination algorithms and adapt their transmit parameters by using appropriate distributed algorithms that exercise control over the radio's operating frequency, time duty cycle or power level [4]. Hidden 


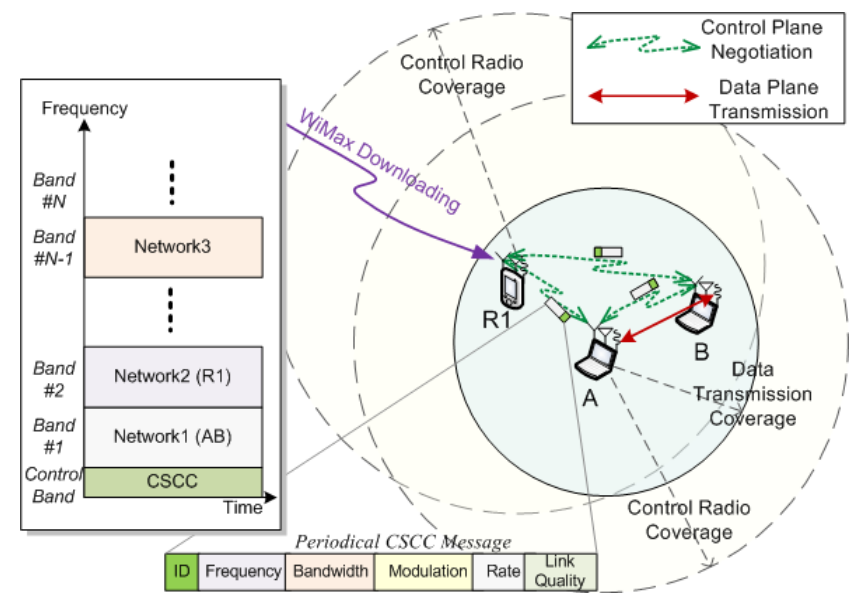

Figure 2. The CSCC spectrum coordination protocol.

node problem can be overcome if the control radio coverage is several times wider than the typical data-path radio coverage (i.e., lower-rate, higher power robust modulation schemes), as shown in Fig. 2. In our prior work, we have studied co-existence between IEEE 802.11b and Bluetooth using an experimental prototype setup [3], demonstrating 30$40 \%$ performance gains. We have also applied CSCC to hypothetical co-existence scenarios with WiFi and WiMax radios sharing the same unlicensed band [1][4], again identifying significant capacity gains relative to simpler reactive schemes.

In the following section, we will introduce three spectrum coordination algorithms designed to operate on top of CSCCbased protocols for the coordination between multiple radios, addressing inter-node and intra-node interference problems.

\section{SPECTRUM COORDINATION ALgORITHMS}

CSCC creates spectrum awareness by allowing information exchange over a common control channel. We designed three coordination algorithms that are based on the information obtained from the CSCC protocol: Bluetooth (BT) Defers Transfer, Bluetooth (BT) Rate Backoff, and SIR-based Coordination. These algorithms define rules or procedures each device has to follow and the CSCC protocol itself is used to carry the shared information between different wireless platforms in a distributed way. To mediate the discussion and lead to evaluations, we tailor the coordination algorithms for an IEEE $802.11 \mathrm{~b} / \mathrm{g}$ and Bluetooth dual-radio platform, which is among the most commonly found multi-radio platform in today's laptops.

\section{A. BT Defer Transfer (Bo)}

Let us consider the multi-radio scenario shown in Fig. 3, where each device is equipped with one IEEE $802.11 \mathrm{~b} / \mathrm{g}$ radio and one Bluetooth radio. The CSCC protocol can be run over a simplified-radio (e.g. operating at $900 \mathrm{MHz}$ band) and exchanges the control packet specified in Fig. 3 periodically. We emulate this common radio channel in our ORBIT experiments by using a low bandwidth logical channel over wired Ethernet connections. Each device exchanges its (data)

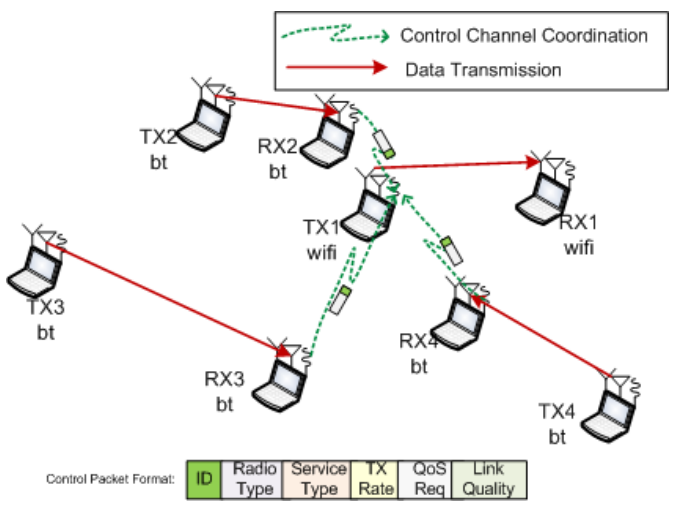

Figure 3. CSCC protocol in an IEEE 802.11g and Bluetooth coexistence scenario.

radio parameters using the CSCC protocol to allow for coordination of transmissions.

The simple algorithm to avoid interference is to allow radios to reserve the channel for duration of a session, granularity of which is on the order of seconds. Specifically in our case, Bluetooth radios (low rate, preempt-able) would avoid WiFi radios' (high rate, preferred) communication to reduce interference. When a Bluetooth device receives a periodic CSCC control message indicating an ongoing WiFi reception, be it from its neighbors or involving its own platform, the Bluetooth transmitter will be turned off during the session when WiFi receiver is active. The underlying conservative assumption with this algorithm is that any Bluetooth transmission will prevent proper $\mathrm{WiFi}$ reception. The variation on this on-off scheme is called BT Rate Back-off and explained in the following subsection.

\section{B. BT Rate Backoff (Rt)}

As an extension to the BT defers transfer (Bo) algorithm, Bluetooth radios can be allowed to throttle their application traffic generation rate to reduce interference to $\mathrm{WiFi}$, while still providing a certain level of QoS to Bluetooth applications. This proposed rate-backoff algorithm allows Bluetooth transmitters to control their channel occupancy in a cooperative and distributed way. The CSCC protocol can overcome the hidden node problem [4] as each node explicitly announces operating parameters in its active state. Each Bluetooth transmitter calculates and enforces appropriate application rate

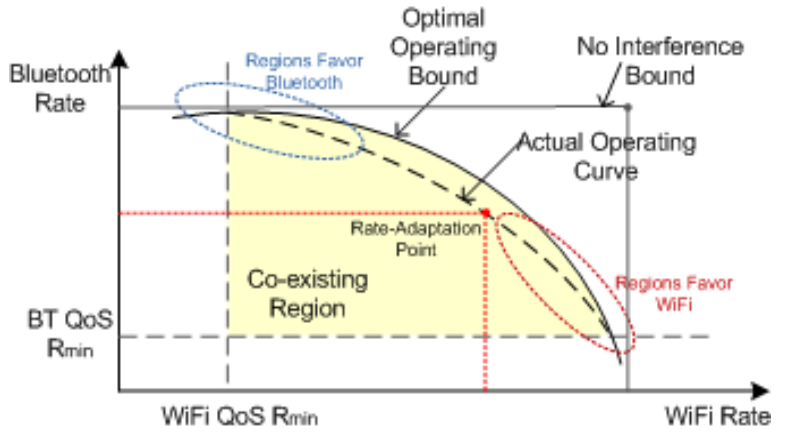

Figure 2. Coexistence Region of Operation for BT-Wifi. 


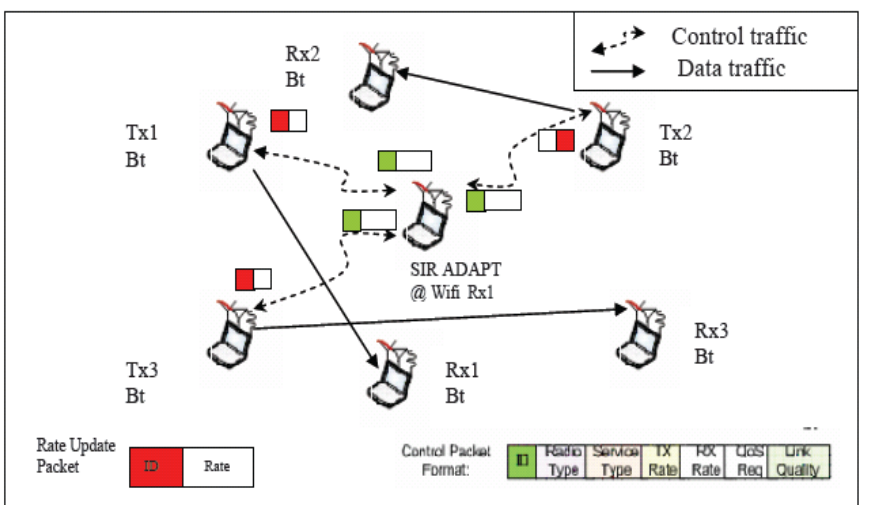

Figure 5. SIR-BT Rate Adaptation for Wifi/BT Coexistence.

(through application demand throttling) based on the information from the control channel. As more WiFi reception activity is observed in the vicinity, Bluetooth radio will choose to lower its channel use by limiting service to application-generated packets in the hope of avoiding hidden receiver [4] activities. We only consider backoff for Bluetooth transmitters in this study and plan to investigate more than one degree of freedom (WiFi and other radios also throttling dynamically) in our future work.

In particular, we assume Bluetooth has audio type CBR traffic with different levels of QoS requirements, with streaming rates from $64 \mathrm{Kbps}$ up to $1 \mathrm{Mbps}$ and WiFi has CBR (25Mbps-Offered load) random on/off type data session. Each time a new neighborhood $\mathrm{WiFi}$ reception activity is identified using CSCC protocol; the Bluetooth transmitter reduces its streaming rate by one level until reaching the lowest level. To address the interference in the same platform, a co-located WiFi radio reception will always force the Bluetooth transmitter to use its lowest service rate (64kbps in our case). When there is no $\mathrm{WiFi}$ receiver detected, the Bluetooth transmitter will work in the highest service rate to make the most use of the available channel.

Figure 4 shows the hypothetical coexistence region defined as the feasible set of (total) Bluetooth and $\mathrm{WiFi}$ service rates for a given network topology. Any given CSCCbased coordination algorithm would aim to support operation along the upper contour of this region, and try to balance Bluetooth and WiFi throughput for satisfying service-level performance criteria. Simple priority schemes, for example backing off Bluetooth rate when WiFi is detected, will tend to position system performance at the bottom right, while favoring Bluetooth will push the operating point to the top left as shown in Fig. 2. More complex strategies can be devised to achieve the desired balance between Bluetooth and WiFi performance, ideally achieving satisfactory level of QoS for each technology. We investigate one such strategy in the following subsection.

\section{SIR-based Coordination}

Signal to Interference Ratio (SIR) based coordination scheme is another CSCC based approach where service rate adaptation at a transmitter is based on the calculated SIR levels of the neighboring receivers. The overall objective of rate adaptation here is to allow interference only if the receivers-under-interference could tolerate it. This ensures successful receptions while considering the minimum QoS requirements at every node in the network. SIR observed at each receiver is theoretically calculated using the information shared by the neighboring transmitters over CSCC. An example scenario of SIR-BT adaptation is shown in Fig. 5 where a WiFi receiver evaluates nearby Bluetooth transmitter activities. The coordination information exchanged from nearby transmitters is the same as in rate backoff algorithm discussed above.

Using the information obtained through CSCC such as the service rate, distances of the transmitters and radio types, the interference caused by neighboring Bluetooth transmitters can be calculated at any $\mathrm{WiFi}$ receiver. With the aggregated information from all interfering transmitters, the total interference and hence the average SIR can then be calculated at those WiFi receivers. Similar to a closed loop control mechanism, the optimum application service rate for co-existence at each Bluetooth transmitter can then be enforced by comparing this calculated SIR with an objective SIR budget. Neighboring Bluetooth transmitters are then updated by WiFi receivers with respective optimum service rates using the rate update packet as shown in Fig. 5. This process can be summarized with the SIR-BT objective function and the set of constraints associated with it as shown in the adjacent SIR-BT algorithm.

In the algorithm, $N_{B T}$ or $N$ is the number of active Bluetooth transmitters detected by WiFi receiver $j$. $\mathrm{I}_{\mathrm{j}}$ is the total interference contributed (theoretically computed) by all the Bluetooth transmitters operating at $R_{i}$, where $R_{i}$ is the application service rate at each of $i$ Bluetooth interferers (transmitters). $I_{i j}$ is the interference contributed by Bluetooth interferer ' $i$ ' at WiFi receiver ' $j$ ' calculated using ' $P_{i}$ ' Bluetooth transmission power, path loss model ' $l p$ ', distance $d_{i j}$, transmission rate $R_{t}$ and service rate $R_{i}$. In Orbit experimental setup, $I_{i j}$ can thus be computed

$$
\begin{aligned}
& \text { While }(\operatorname{SIRj}(\vec{R})<\text { SIR_linkbudget) } \\
& \{ \\
& \text { Maximize } \operatorname{SIRj}(\vec{R}) \text { where } \\
& S I R_{j}=\left(P_{t}-l p\right)-I_{j}(\vec{R}) \\
& I_{j}(\vec{R})=10 \log \left(\sum_{i=1}^{N} I_{i j}\left(R_{i}\right)\right) \\
& I_{i j}=10^{\left[\left(P_{i}-l p\left(d_{i j}\right)+10 \log \left(R_{i} / R_{t}\right)\right) / 10\right]} \forall i=1,2,3 \ldots . N \\
& \operatorname{lp}\left(d_{i j}\right)=40+10 * \alpha * \log \left(d_{i j}\right) \text { where } \ldots \alpha=2 \\
& \text { Subject to: } \\
& R_{\text {max }}\left(\text { Qos } s_{-} \text {Max }\right)>=R_{i}>=R_{\text {min }}(\text { Qos _ Min }) \\
& \text { where... } \vec{R}=\left[R_{i}\right]_{1 \times N_{B T}} \forall i=1,2,3 \ldots N_{B T} \\
& \text { \} }
\end{aligned}
$$




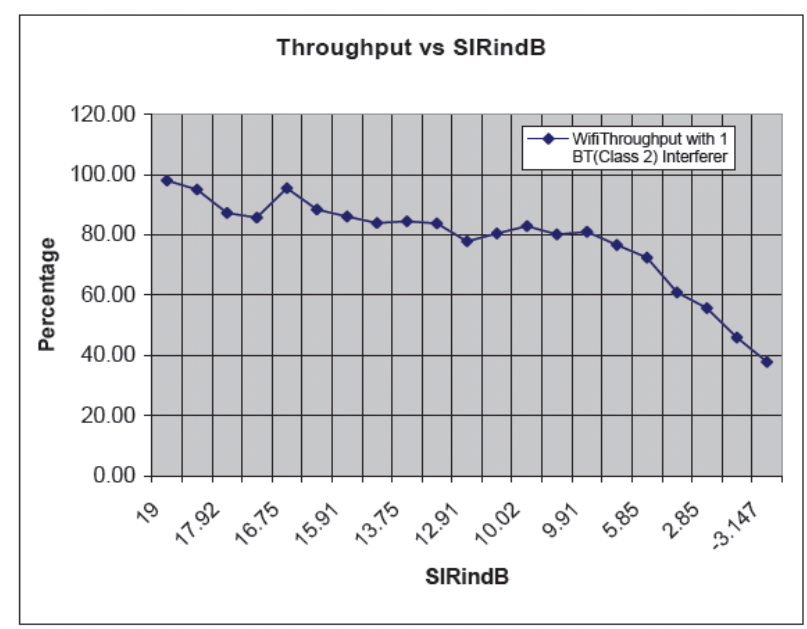

Figure 6. Throughput vs SIR curve for $802.11 \mathrm{~g}$ with Bluetooth (Class 2) Interferer.

theoretically using known distances $d_{i j}$, but in real world only a rough estimate of $I_{i j}$ is possible using the RSSI values at the control channel. Since distance $d_{i j}$ of the interferer and path loss constant cannot be determined in real world setups, the transmitters, which ensures $S I R_{j}$ meets the SIR link budget constraint, is calculated and communicated back to Bluetooth.

In particular for our initial experimental evaluations, we empirically studied the SIR link budget which would allow WiFi receivers maintain an acceptable level of throughput without much performance degradation. With IEEE 802.11 link adaptation mechanism enabled (i.e. Sample Rate for our wireless device drivers), our WiFi (IEEE 802.11g) and Bluetooth (class 2) dual radio experiments revealed that $10 \mathrm{~dB}$ of an SIR budget at WiFi receivers resulted in a worst case maximum of $25 \%$ deviation from the throughput that was achievable in the absence of any interference, as illustrated in Fig. 6. This average is also in agreement with the theoretical IEEE $802.11 \mathrm{~g}$ SIR budget range of approximately $4 \mathrm{~dB}$ to $22 \mathrm{~dB}$ for $6 \mathrm{Mbps}$ and $54 \mathrm{Mbps}$ respectively.

SIR-BT adaptation approach ensures an optimum WiFi performance by maintaining an SIR $>=S I R \_l i n k b u d g e t$ at the WiFi receivers and also invokes a need-based Bluetooth rate adaptation depending on the interference levels observed at the receiver. As we will detail in the following section, this approach shows throughput improvements in all scenarios we have tested. The same

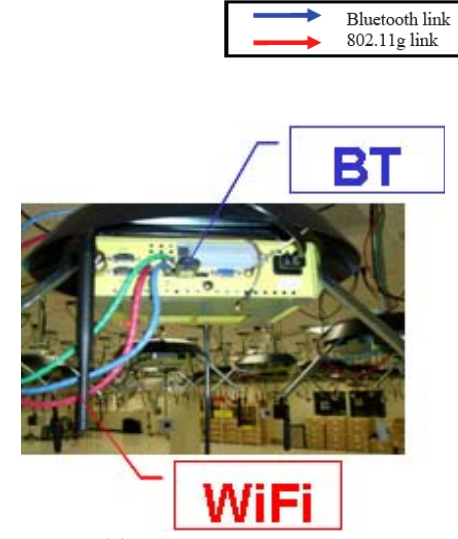

(a)

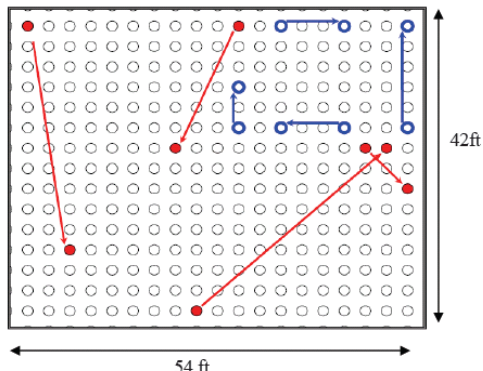

(d)

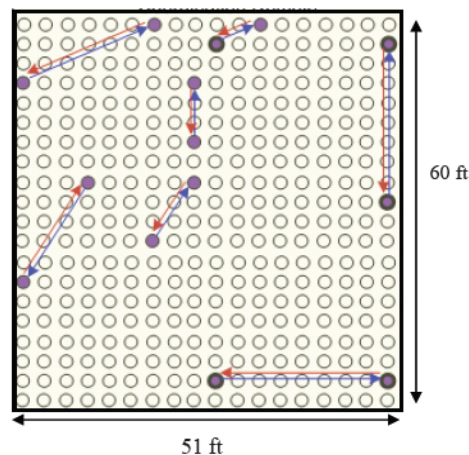

(b)

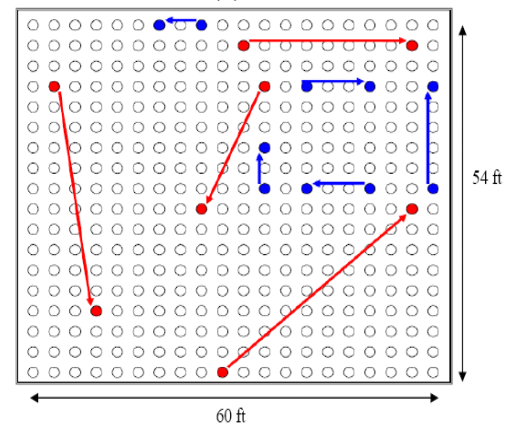

(e)

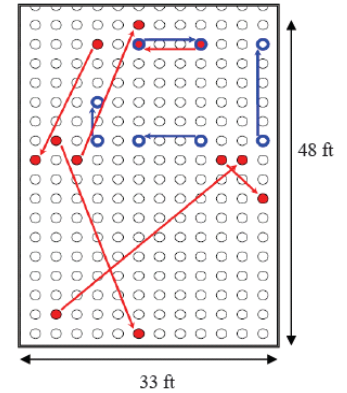

(c)

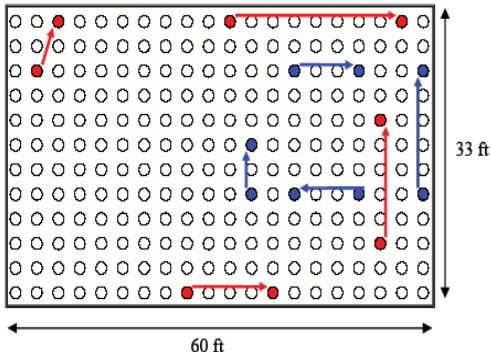

(f)

Figure 7. Experiment environment. (a) ORBIT multi-radio node (b - f) evaluation topologies 1 through 5.

proposed SIR-BT design gives an approximate upper bound for any real world SIR based scheme. Thus the $S I R_{j}$ at each WiFi receiver is computed and an optimum $\vec{R}$, the vector representing service rates of all the $N_{B T}$ active Bluetooth scheme can also be extended to include WiFi transmitters into the control loop and is being investigated as our ongoing work. 


\section{EXPERIMENTAL EVALUATION ON THE ORBIT TESTBED}

\section{A. Experiment Setup}

We conducted experiments on the ORBIT testbed [12][14] with a total of 400 multi-radio wireless nodes deployed in a dense setting (i.e. an average of 3-feet inter-node distances) to evaluate our adaptation strategies. The WiFi - Bluetooth dual radio node used in the ORBIT experiments is shown in Fig. 7(a), where the physical separation of about a foot between the Bluetooth USB dongle and the WiFi antenna can be seen. Radio and traffic parameters for the experiments are summarized in Table I. Figure 7(b)-(f) illustrates the specific co-existence topologies we have evaluated on the testbed, using up to 16 radios for most of the experiments (up to 22 radios were used for the overhead evaluation in subsection $D$ ). For the topology given in Fig. 7(b), both class 1 and class 2 Bluetooth radios and IEEE $802.11 \mathrm{~g}$ radios are tested for intranode interference and inter-node interference, when Bluetooth communication flows from node1 to node2, $\mathrm{WiFi}$ communication flows in the opposite direction, from node2 to node1, representing the worst-case interference scenario. Fig. 7(c)-(f) are the topologies with class- 2 only Bluetooth and IEEE $802.11 \mathrm{~g}$ radios; they are used to evaluate inter-node interference due to multiple interferers in proximity. For the WiFi traffic, we consider random ON/OFF type CBR sessions with randomized session intervals and for the Bluetooth traffic CBR type audio streams with different service levels are used, as detailed in Table I.

TABLE I. ORBIT EXPERIMENT PARAMETER

\begin{tabular}{|c|c||c|}
\hline & \multicolumn{2}{|c|}{ Data Radio Service } \\
\hline PHY Type & IEEE 802.11g & Bluetooth (Belkin / IOgear \\
(Atheros AR5212) & USB Dongle) \\
\hline Frequency & $2427-2447 \mathrm{MHz}$ & $2402-2483.5 \mathrm{MHz}$ \\
\hline Modulation & OFDM (256 FFT) & GFSK + FHSS (DQPSK for \\
& QAM & EDR) \\
\hline Transmit Power & $18 \mathrm{dBm}$ & $4 \mathrm{dBm}(\sim 20 \mathrm{~m})$ (class 2) \\
& & $20 \mathrm{dBm}(\sim 100 \mathrm{~m})$ (class 1) \\
\hline PHY Rate & Up to 54Mbps & Upto 1Mbps (class 2) \\
& AutoRate and Fixed & Upto 2.1Mbps (class 1 w/ \\
& Rate 36Mbps & EDR) \\
\hline Data session & Pareto ON/OFF & Constant bitrate audio \\
& variable duration & streaming \\
& CBR: 5 sec random & $(64,128,320,512,1024 \mathrm{Kbps})$, \\
& session, 1 KB packet & Packet Size of 1 KB \\
\hline
\end{tabular}

B. Experimental results for BT Defer transfer (Bo) and BT rate backoff (Rt) schemes with intra and inter node interference

Average throughput measurements from the evaluation of the topology given in Fig. 7(b) with $104 \mathrm{Kbps}$ Bluetooth offered load and varying WiFi loads are plotted in Fig. 8. Average session throughput for WiFi under no coordination as well as under $B T$ Defer and BT rate backoff schemes are plotted in Fig.

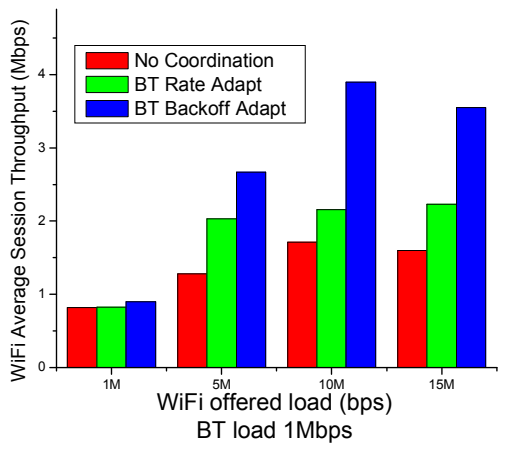

(a)

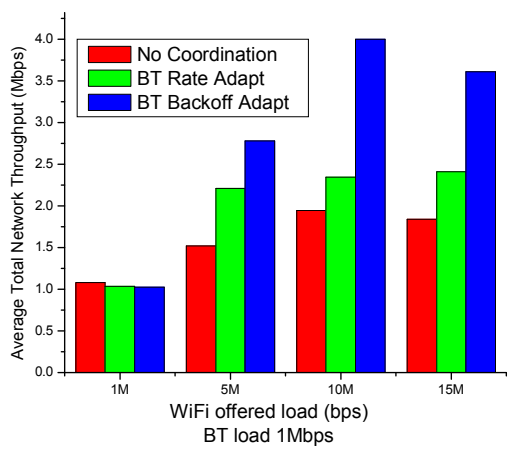

(c)

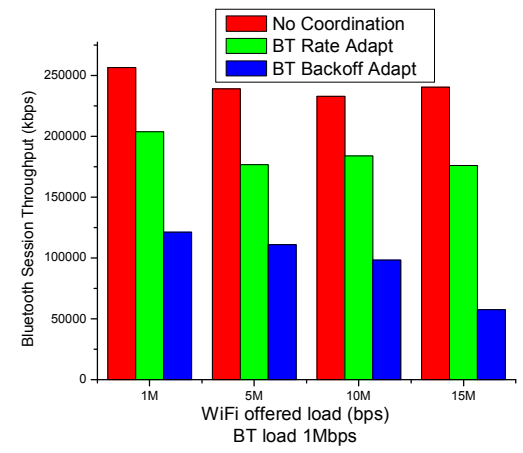

(b)

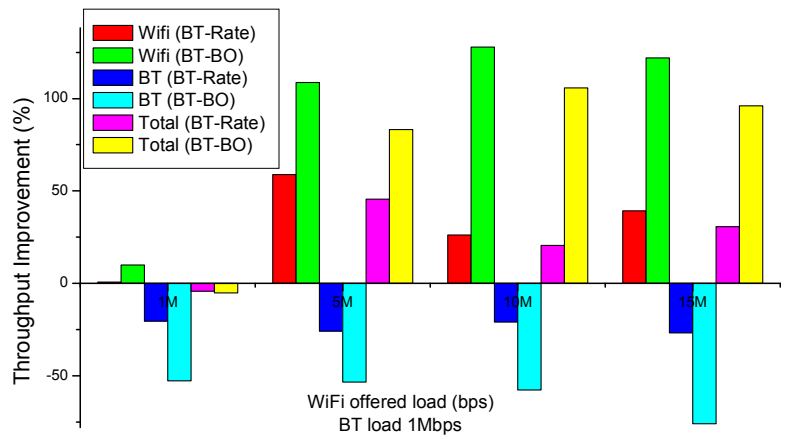

(d)

Figure 8. Experimental results for network throughput vs. WiFi loading rate. (a) WiFi session throughput (b) BT throughput (c) Average total network throughput (d) Throughput improvement for each case. 
8(a). The same case for Bluetooth session throughput is measured and plotted in Fig. 8(b). Also, total network throughput is plotted in Fig. 8(c). Summary of percent throughput gains are given in Fig. 8(d) for all the cases. We observe that BT Defers strategy provides the largest gains for WiFi throughput (over 100\%), more emphasized with increasing $\mathrm{WiFi}$ load levels. However, the penalty to the Bluetooth traffic is significant $-60 \%$ to $80 \%$ loss experienced by Bluetooth audio streaming applications. BT rate backoff finds a better balance between $\mathrm{WiFi}$ and Bluetooth throughput - up to $50 \% \mathrm{WiFi}$ throughput improvements are observed for a maximum loss of up to $20 \%$ in Bluetooth throughput. This motivates the adoption of intelligent adaptation schemes where the desired balance could be found by the appropriate use of the information provided by spectrum aware protocols like CSCC.

\section{Experimental Results for SIR-BT adaptation}

SIR-BT adaptation algorithm outlined in Section $\mathrm{III}(\mathrm{C})$ is evaluated on four different topologies illustrated in Fig. 7(c)-(f), involving up to 16 radios. These topologies help to study the effects of inter-node interference, the overhead involved and the ability to

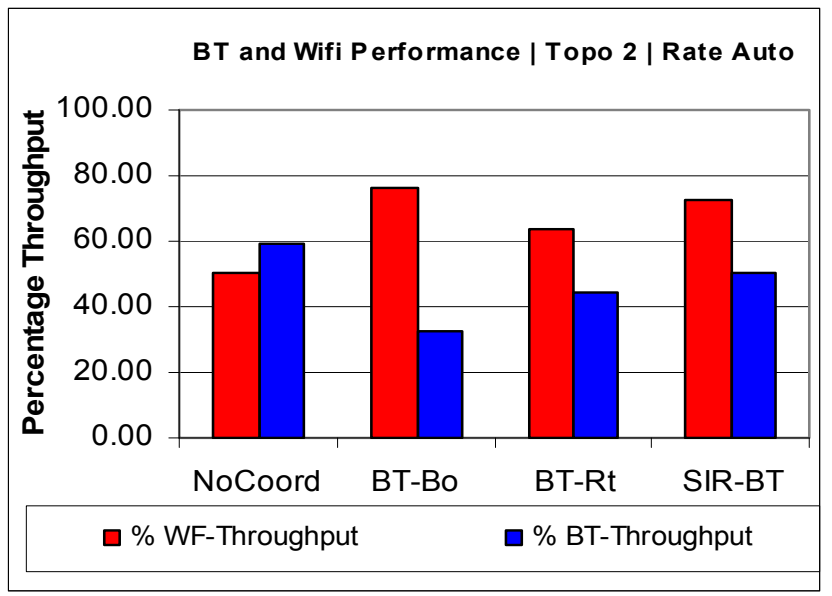

(a)

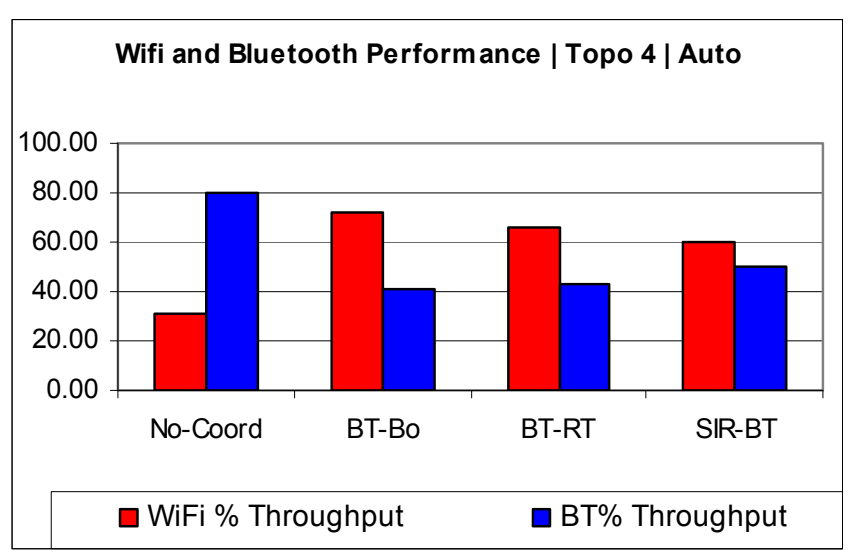

(c) coordinate multiple proximity located devices with minimum or no intra-node interference.

\section{1) Results - UDP Traffic -Throughput loss}

Normalized percent-throughput measurement results are provided in Fig. 9 for SIR-BT scheme. To facilitate easy comparisons, results from no coordination case and application of the earlier two schemes are also shown in the same figure. Normalizations are done with respect to the appropriate single radio network (i.e., WiFi-only or Bluetoothonly) case. Plots in Fig. 9(a)-(d) correspond to the topologies in Fig. 7(c)-(f) respectively. First of the bars (lighter color) in two-bar plots always corresponds to the throughput for WiFi whereas the second of the bars corresponds to the throughput for Bluetooth network.

No Coordination Case: We observe that lack of coordination may cause up to $70 \%$ throughput loss for $\mathrm{WiFi}$, and up to $40 \%$ throughput loss for BT, depending on the particular topology due to interplatform interference as seen from the first pair of bars in Fig. 9(a-d). These significant losses indicate the need for inter-node interference mitigation schemes.

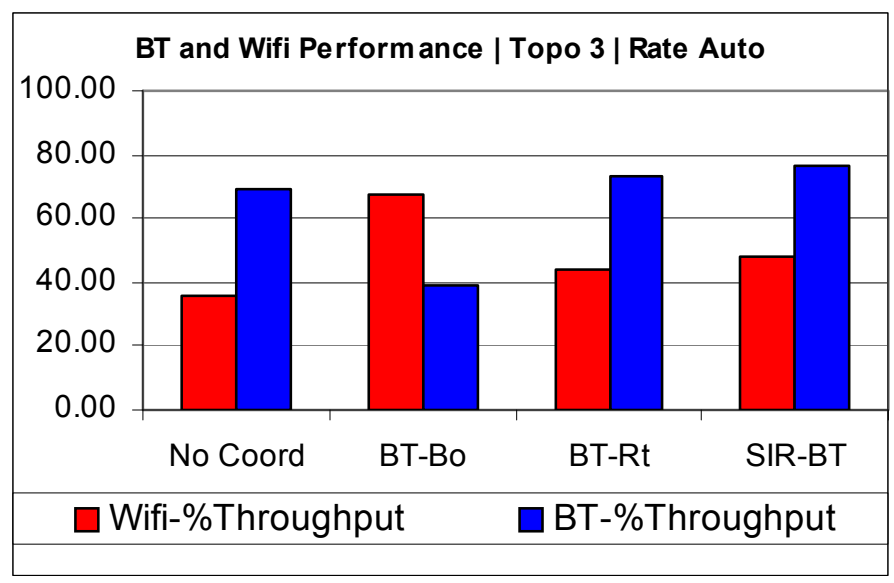

(b)

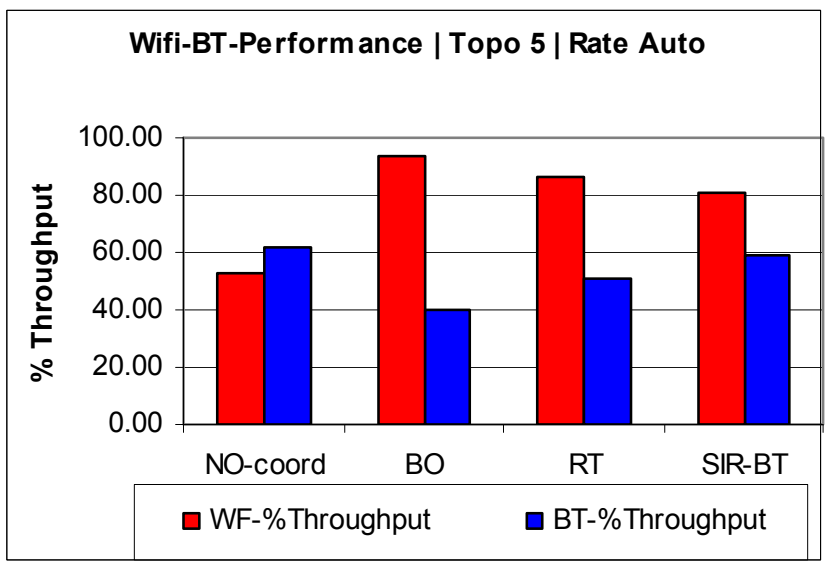

(d)

Figure 9. Results for WiFi-BT throughput vs. Coordination Scheme, (a-d) correspond to topologies in Fig. 7(c-f). 
Bluetooth performs better than WiFi due to some unused frequencies selected during frequency hopping.

Bo, Rt, SIR-BT Case: Similar to the intra-node interference case reported in Section IV(B), BT-Defer scheme here again provides the best throughput recovery for $\mathrm{WiFi}$, while penalizing Bluetooth to greater extents. BT rate backoff addresses this proportionality problem and increases Bluetooth throughput up to $35 \%$ at a reasonable cost to the $\mathrm{WiFi}$ network. SIR-BT scheme further improves Bluetooth throughput up to $10 \%$ more than $B T$ rate backoff scheme with minimal or no degradation in WiFi. SIRBluetooth scheme here operates at SIR link budget threshold value of $10 \mathrm{~dB}$.

\section{2) Result - TCP traffic - File Transfer Delay}

In order to quantify the effect of multi-radio coexistence on everyday applications better, we study a file exchange scenario over TCP protocol. The topology \#4 in Fig. 7(e) was used and a $1 \mathrm{MB}$ data file was exchanged across each WiFi radio link using TCP as the underlying transport protocol. The file transfer times were recorded for each $\mathrm{WiFi}$ link and the average time to complete the transfer is plotted Fig. 10.

No Coordination Case: The file transfer time more than doubles under inter-node interference as shown by the second pair of bars in Fig. 10. This experimentally proves that the download times/file exchange times in real world setups would more than double under such dense multi-radio scenarios.

Bo, Rt, SIR-BT Case: BT Defer (Bo) scheme reduces time to complete to almost no interference scenario at the cost of Bluetooth throughput shown in Fig. 10. We also observe that SIR-BT adaptation finds a better balance between Bluetooth and $\mathrm{WiFi}$ throughput compared to BT RateBackoff scheme, reducing transmission time by another $20 \%$ with almost no compromise in Bluetooth performance as seen from last pair of bars in Fig. 10.

\section{3) Choice and Sensitivity of SIR link budget threshold}

Choice: SIR link budget threshold for WiFi receivers with IEEE 802.11 auto link rate adaptation was empirically determined to be around $10 \mathrm{~dB}$. The same experiment with fixed link rate at $36 \mathrm{Mbps}$ suggests SIR budget to be around $16 \mathrm{~dB}$, in agreement with IEEE 802.11g OFDM decoding requirements. This choice of SIR link budget threshold serves as a handle to decide the point of operation on the coexistence curve shown in Fig. 4.

Sensitivity: To study the sensitivity of the threshold value, we tested the topology \#4 with

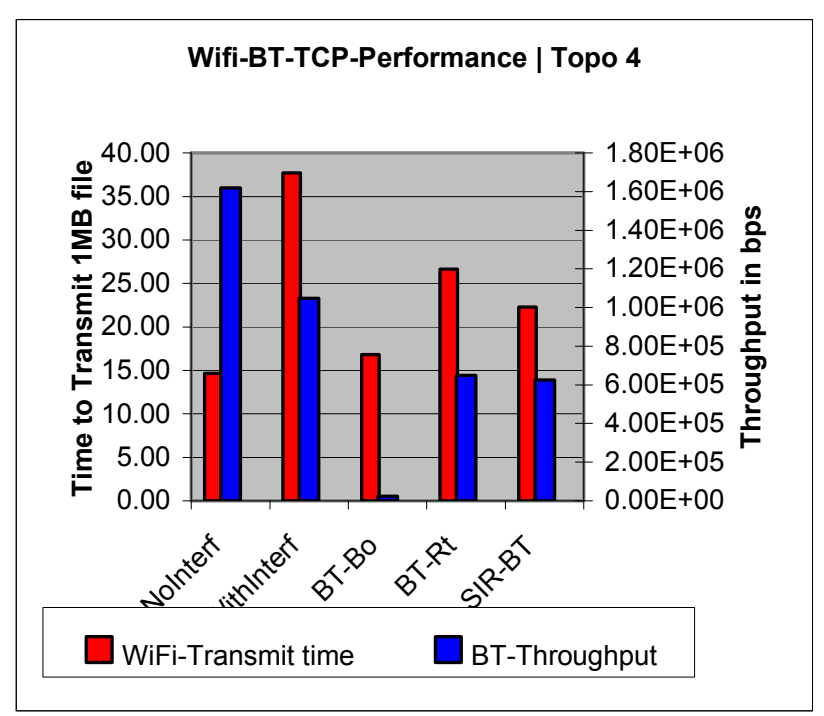

Figure 10. WiFi-g-1Mbyte TCP file transfer time and BT throughput in Topology 4.

different SIR threshold values (around $16 \mathrm{~dB}$ for fixed transmission mode at 36Mbps) for SIR-9.5, 14, 16, 19 and 22 as shown in Fig. 11. Experimental results confirm low sensitivity to exact values of the SIR budget, thus making practical implementations for adaptation feasible.

\section{Estimating Control Overhead}

The overhead involved in our adaptation approach may be considered in terms of the memory and the bandwidth requirements of the control data exchange through CSCC protocol. For $N$ different type of radios in the environment $(N=2$ for our dual $\mathrm{WiFi}-$ Bluetooth example setup), CSCC requires $11+16^{*} N$ bytes of periodic information dissemination from each radio every second. In Table II, we calculated the payload

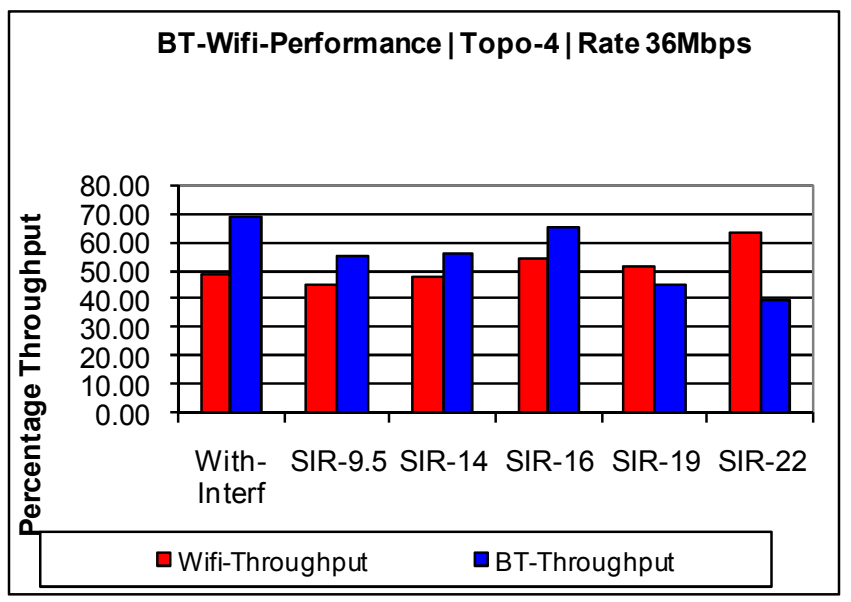

Figure 11. SIR-BT scheme with Fixed link Rate at 36Mbps for multiple SIR budget threshold values. 
TABLE II.

OVERHEAD FOR THE ADAPTATION SCHEMES

\begin{tabular}{|c|c|c|c|c|c|c|}
\hline \multirow[b]{3}{*}{$\begin{array}{c}\text { \# of radio } \\
\text { nodes } \\
\text { K }\end{array}$} & \multirow{2}{*}{\multicolumn{2}{|c|}{$\begin{array}{c}\text { Control Payload Size(CSCC } \\
\text { Packet) }\end{array}$}} & \multirow{3}{*}{$\begin{array}{c}\begin{array}{c}\text { Total Control } \\
\text { Data per } \\
\text { second }\end{array} \\
\text { B in bps }\end{array}$} & \multicolumn{2}{|c|}{ Throughput } & \multirow{3}{*}{$\begin{array}{c}\begin{array}{c}\text { Payload } \\
\text { Overhead } \\
\text { ratio (\%) }\end{array} \\
\text { B/(Y-X) }\end{array}$} \\
\hline & & & & \multirow{2}{*}{$\begin{array}{c}\begin{array}{c}\text { Without } \\
\text { Coordination }\end{array} \\
\begin{array}{c}\text { No } \\
\text { Coordination } \\
(\mathrm{X} \text { in bps })\end{array}\end{array}$} & \multirow{2}{*}{$\begin{array}{c}\text { With } \\
\text { Coordination } \\
\text { Bluetooth } \\
\text { Backoff } \\
\text { Coordination } \\
(Y \text { in bps }) \\
\end{array}$} & \\
\hline & $\begin{array}{l}\text { Fixed Part } \\
11 \text { bytes }\end{array}$ & $\begin{array}{l}\text { Variable Part } \\
16 * N \text { bytes } \\
(\mathrm{N}=2 \text { for dual } \\
\text { radio setup) }\end{array}$ & & & & \\
\hline 8 & $88 \mathrm{~B}$ & $256 \mathrm{~B}$ & 2752 & $1.59 \mathrm{E}+07$ & $2.45 \mathrm{E}+07$ & 0.03 \\
\hline 16 & $176 \mathrm{~B}$ & $512 B$ & 5504 & $1.11 \mathrm{E}+07$ & $2.26 \mathrm{E}+07$ & 0.05 \\
\hline 22 & 242B & 704B & 7568 & $1.6 \mathrm{E}+07$ & $2.17 \mathrm{E}+07$ & 0.13 \\
\hline
\end{tabular}

overhead for different size networks of $\mathrm{K}$ radio nodes, and compared it with the gains obtained from the adaptation to find the overhead ratio. The overhead introduced by the adaptation approaches is very small ( $\sim 0.1 \%$ for $\sim 20$ nodes) and should be acceptable for system design.

\section{CONCLUSIONS AND FUtURE WORK}

In this paper we have experimentally studied the spectrum coexistence problems of multi-radio platforms in a dense radio environment. We have experimentally proved that in dense scenarios similar to topologies $\# 1-\# 5$, the interference from neighboring radios is severe leading to drop in WiFi performance by a factor of $3 \mathrm{x}-4 \mathrm{x}$ and by $1.5 \mathrm{x}-2 \mathrm{x}$ in the case of Bluetooth. As a case study, we designed, implemented and evaluated three adaptation schemes for a dualradio (WiFi and Bluetooth) scenario with different topologies, where CSCC spectrum etiquette protocol is used as a mechanism to allow spectrum coordination in a distributed way. Our evaluations show that $B T$ defers scheme can improve the total network throughput by $30-100 \%$ at the cost of Bluetooth performance. The BT-Rate Backoff scheme achieves a slightly better operating point for both radios, with a moderate $20 \%$ degradation for Bluetooth throughput and $50 \%$ improvement for $\mathrm{WiFi}$ throughput. The SIR$B T$ scheme can improve Bluetooth throughput by an additional factor of $8-10 \%$ and WiFi network by $20 \%$ when compared to the BT-Rate Backoff scheme, thus achieving a more balanced operating point for Bluetooth-WiFi dual-radio interference scenarios.

Further work planned includes augmenting the ORBIT testbed with WiMax and UWB radios to fully evaluate the SOHO scenario, and to evaluate enhanced versions of BT Rate Back-off and SIR-BT schemes.

\section{REFERENCES}

[1] X. Jing, S. Mau, D. Raychaudhuri and R. Matyas, "Reactive Cognitive Radio Algorithms for Co-Existence between IEEE
$802.11 \mathrm{~b}$ and 802.16a Networks," In Proc. of IEEE Globecom 2005, Nov. 28-Dec. 2, 2005.

[2] J. Zhu, A. Waltho, X. Yang, X. Guo, "Multi-Radio Coexistence: Challenges and Opportunities", In Proc. of IEEE ICCCN 2007.

[3] D. Raychaudhuri and X. Jing, "A spectrum etiquette protocol for efficient coordination of radio devices in unlicensed bands," In Proc. of IEEE PIMRC 2003, Sept. 2003.

[4] X. Jing and D. Raychaudhuri, "Spectrum Co-existence of IEEE 802.11b and 802.16a Networks using the CSCC Etiquette Protocol," In Proc. of IEEE DySPAN 2005, Nov. 8-11, 2005.

[5] D. P. Satapathy and J. M. Peha, "Performance of Unlicensed Devices With a Spectrum Etiquette," in Proc. of IEEE Globecom, Nov. 1997.

[6] D. P. Satapathy and J. M. Peha, "Etiquette Modification for Unlicensed Spectrum: Approach and Impact," in Proc. of the IEEE Vehicular Technology Conference, vol. 1, May 1998.

[7] C.F. Chiasserini, R. Rao, "Performance of IEEE 802.11 WLANs in a Bluetooth Environment," in Proc. of IEEE Wireless Communications and Networking Conference, WCNC 2000, Sept. 2000.

[8] N. Glomie, R. E. Van Dyck, A. Soltanian, "Interference of Bluetooth and IEEE 802.11: Simulation Modeling and Performance Evaluation," in Proc. of the Fourth ACM International Workshop on Modeling,Analysis and Simulation of Wireless and Mobile Systems, MSWIM'01, July 2001.

[9] A Kamerman, "Coexistence between Bluetooth and IEEE 802.11 CCK Solutions to Avoid Mutual Interference," presentation to Lucent Technologies Bell Laboratories, Jan. 1999.

[10] M. Hnnikinen, T. Rantanen and J. Ruotsalainen, "Coexistence of Bluetooth and Wireless LANs," in Proc. of the 8th edition of IEEE ICT, 2001.

[11] G. Ennis, "Impact of Bluetooth on 802.11 Direct Sequence," in IEEE P802.11 Working Group Contribution, IEEE P802.1198/319, Sept. 1998.

[12] D. Raychaudhuri, "ORBIT: Open-Access Research Testbed for Next-Generation Wireless Networks," NSF Network Research Testbeds Program, NSF award \#ANI-0335244, 2003-07, May 2003.

[13] A. Ergin, K. Ramachandran, and M. Gruteser, "Understanding the Effect of Access Point Density on Wireless LAN Performance," in ACM MOBICOM 2007, 350-353, Sept. 2007.

[14] ORBIT radio grid testbed, www.orbit-lab.org 